\title{
The effect of hematocrit during hypothermic cardiopulmonary bypass in infant heart surgery: Results from the combined Boston hematocrit trials
}

David Wypij, PhD, ${ }^{\text {a,d,h }}$ Richard A. Jonas, MD, ${ }^{\text {be, }, *}$ David C. Bellinger, PhD, MSc, ${ }^{\text {c,f }}$ Pedro J. Del Nido, MD, ,e John E. Mayer, Jr., MD, , ${ }^{\text {,e }}$ Emile A. Bacha, MD, ${ }^{\text {,e }}$ Joseph M. Forbess, MD, ${ }^{\text {be, }}{ }^{\dagger}$ Frank Pigula, MD, ,e

Peter C. Laussen, MD, and Jane W. Newburger, MD, $\mathrm{MPH}^{\mathrm{a}, \mathrm{d}}$

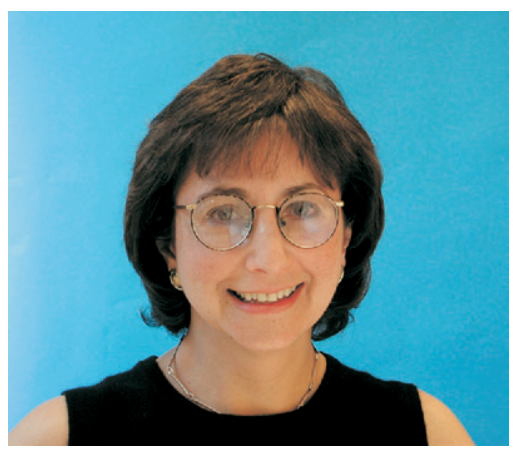

Dr Newburger

See related articles on pages 240 and 347.

From the Departments of Cardiology, ${ }^{\text {a }}$ Cardiovascular Surgery, ${ }^{\mathrm{b}}$ and Neurology, ${ }^{\mathrm{c}}$ Children's Hospital Boston; the Departments of Pediatrics, ${ }^{\mathrm{d}}$ Surgery, ${ }^{\mathrm{e}}$ Neurology, ${ }^{\mathrm{f}}$ and Anesthesia, ${ }^{\mathrm{g}}$ Harvard Medical School; and the Department of Biostatistics, ${ }^{\mathrm{h}}$ Harvard School of Public Health, Boston, Mass.

Registered with clinicaltrials.gov (\#) NCT00006183.

Supported by grants HL 063411 and RR 02172 from the National Institutes of Health and by the Farb Family Fund.

Received for publication Jan 9, 2007; revisions received Feb 9, 2007; accepted for publication March 6, 2007.

Address for reprints: Jane W. Newburger, MD, MPH, Department of Cardiology, Children's Hospital Boston, 300 Longwood Ave, Boston, MA 02115 (E-mail: jane. newburger@cardio.chboston.org).

*Dr Jonas is currently Chief of Cardiovascular Surgery and Co-Director of the Children's National Heart Institute, Washington, DC.

$\dagger$ Dr Forbess is currently at Children's Medical Center Dallas.

J Thorac Cardiovasc Surg 2008;135:355-60

0022-5223/ $\$ 34.00$

Copyright (C) 2008 by The American Association for Thoracic Surgery

doi:10.1016/j.jtcvs.2007.03.067
Objective: Two randomized trials of hematocrit strategy during hypothermic cardiopulmonary bypass in infant heart surgery have been performed. The first suggested worse outcomes were concentrated in patients with lower hematocrit levels (approximately 20\%), whereas the second suggested there was little benefit to increasing the hematocrit level above $25 \%$. The form of the relationship between continuous hematocrit levels and outcomes requires further study.

Methods: In the two trials, 271 infants who underwent biventricular repair not involving the aortic arch were enrolled. Analysis was undertaken of the effects of hematocrit level, as a continuous variable, at the onset of low-flow cardiopulmonary bypass.

Results: Psychomotor Development Index scores at age 1 year varied nonlinearly with hematocrit levels, with increasing scores up to $23.5 \%$ hematocrit $(P<.001)$ and a plateau effect beyond $23.5 \%(P=.42)$, based on a piecewise linear model. Lower hematocrit levels were associated with more positive intraoperative fluid balance $(P<.001$ for linear trend) and marginally associated with higher serum lactate levels at 60 minutes after bypass $(P=.08$ for linear trend), but not with blood products given, nadir of cardiac index in the first 24 hours, or Mental Development Index scores.

Conclusions: A hematocrit level at the onset of low-flow cardiopulmonary bypass of approximately $24 \%$ or higher is associated with higher Psychomotor Development Index scores and reduced lactate levels. Because the effects of hemodilution may vary according to diagnosis, age at operation, bypass variables such as $\mathrm{pH}$ strategy and flow rate, and other perioperative factors, this study cannot ascertain a universally "safe" hemodilution level.

$\mathrm{H}$ emodilution during hypothermic cardiopulmonary bypass (CPB) was used initially in the 1950s, with the rationale that it would reduce the use of blood and blood products, ${ }^{1}$ as well as improve perfusion in microvessels. ${ }^{2}$ Subsequently, concerns emerged about potential adverse effects of hemodilution, including reduced perfusion pressure, increased cerebral blood flow and hence increased risk for cerebral microemboli, and decreased oxygen-carrying capacity. ${ }^{3-5}$

$\mathrm{We}^{6}$ previously conducted a randomized trial of 147 patients, of whom 74 were assigned to a lower-hematocrit strategy at onset of low-flow CPB $(21.5 \% \pm 2.9 \%$, mean \pm SD, with a target of $20 \%)$ and 73 to a higher-hematocrit strategy $(27.8 \% \pm$ $3.2 \%$, with a target of $30 \%$ ). The lower-hematocrit group had higher intraoperative fluid balance, higher serum lactate levels 60 minutes after cessation of CPB, lower nadirs of cardiac index in the first 24 hours, and, at age 1 year, worse Psychomotor Development Index (PDI) scores, although Mental Development Index (MDI) scores were similar between groups. Because we could not determine a specific 

Abbreviations and Acronyms
$\mathrm{CI}=$ confidence interval
$\mathrm{CPB}=$ cardiopulmonary bypass
D-TGA $=$ dextro-transposition of the great arteries
MDI = Mental Development Index
PDI = Psychomotor Development Index
TOF $=$ tetralogy of Fallot
VSD $=$ ventricular septal defect

target hematocrit level that was optimal, we embarked on a second randomized trial of target hematocrit $25 \%$ versus $35 \%$ in a similar study population.

A companion article $^{7}$ presents results from an intent-totreat analysis of 124 patients, of whom 56 were assigned to a medium-hematocrit strategy at onset of low-flow CPB $(24.8 \% \pm 3.1 \%$, with a target of $25 \%)$ and 68 to a higherhematocrit strategy $(32.6 \% \pm 3.5 \%$, with a target of $35 \%)$. These two treatment groups had similar serum lactate levels 60 minutes after cessation of CPB, nadirs of cardiac index in the first 24 hours, PDI scores, and MDI scores, although the $25 \%$ hematocrit group had higher intraoperative fluid balance and, only in a subgroup with dextro-transposition of the great arteries (D-TGA), longer hospital stay. We concluded that hemodilution to hematocrit levels of $35 \%$ compared with $25 \%$ had marginal clinical benefits and no increased risks.

In this article, we combine the data from these two trials to attempt to identify, in more quantitative terms, the relationship of hemodilution level to key postoperative and 1-year developmental outcomes.

\section{Patients and Methods}

The protocols for both hematocrit trials have been described previously ${ }^{6,7}$ and were similar except for the targeted hemodilution levels. We enrolled patients between November 1996 and July 2004 at Children's Hospital Boston. Eligibility criteria are summarized elsewhere and were identical in both trials. ${ }^{6,7}$ In brief, we included infants under age 9 months with congenital heart lesions comprised of two ventricles without aortic arch obstruction, absence of suspected or definite genetic disorders or congenital anomalies of more than minor severity, and no previous or planned cardiac surgery before the 1-year developmental evaluation. Parental informed consent was obtained according to institutional guidelines.

Of 424 eligible infants for whom informed consent was requested, 278 patients were enrolled; of these, 7 (3\%) were excluded post hoc because the operation was not performed as planned or because the patient was discovered to have been ineligible after further clinical information was obtained. The remaining 271 patients underwent reparative surgical intervention according to the study protocols $\mathrm{s}^{6,7}$ and constitute the study population. Full-flow CPB $\left(\sim 2.5 \mathrm{~L} \cdot \mathrm{min}^{-1} \cdot \mathrm{m}^{-2}\right)$ was used during cooling and rewarming. Some patients had periods of deep hypothermic circulatory arrest, and most had at least one period of reduced-flow $\mathrm{CPB}$, for example, at approximately $0.75 \mathrm{~L} \cdot \mathrm{min}^{-1} \cdot \mathrm{m}^{-2}$ when at deep hypothermia (rectal temperature $<18^{\circ} \mathrm{C}$ ). For patients in whom low-flow CPB was not used, we analyzed the hematocrit level 10 minutes after onset of cooling.

Participating infants were randomly assigned to undergo hemodilution to a hematocrit level of approximately $20 \%$ versus $30 \%$ in the first trial and approximately $25 \%$ versus $35 \%$ in the second trial. Treatment assignments were based on randomized blocks with stratification according to surgeon and diagnostic group. The method of support was assigned immediately before the operation. Perfusionists and study nurses were aware of treatment assignment. Surgeons, anesthesiologists, cardiac intensivists, and developmental psychologists were blinded to treatment assignment.

\section{Statistical Analyses}

Outcomes included the perioperative and 1-year development variables that were the primary outcome variables for at least one of the two hematocrit trials. These include serum lactate levels 60 minutes after cessation of CPB, nadir of cardiac index in the first 24 hours, and the PDI and MDI of the Bayley Scales of Infant Development at age 1 year. ${ }^{8}$ We also explored the relationship of hematocrit to fluid balance, use of blood products, and duration of intubation, intensive care unit stay, and hospital stay.

Analyses assessing the effects of hematocrit at the onset of low-flow CPB as a continuous variable on outcomes used linear regression, proportional hazards regression for length-of-stay variables, and generalized additive models. ${ }^{9,10}$ Generalized additive models are methods of nonparametric regression smoothing that allow for the assessment of any effect of hemodilution level on outcome, including nonlinear effects, as well as assessment of departure of linearity or piecewise linearity of effect. Piecewise linear (or cut point) regression models were used to model outcomes that had significant nonlinear trends with hematocrit level. Confidence intervals for cut points were based on inversion of likelihood ratio tests. ${ }^{11}$ All likelihood maximizations and likelihood ratio tests searched over a grid of hematocrit levels in $0.5 \%$ increments. Quadratic regression models were also considered but did not fit the data better than either generalized additive models or piecewise linear models. $\mathrm{We}^{12}$ have used similar methods to assess the effects of duration of deep hypothermic circulatory arrest in infant heart surgery on late neurodevelopment.

All regression analyses adjust for diagnostic group and, if statistically significant, year of operation (coded as 19961998, 1999-2000, 2001-2002, and 2003-2004). We also explored whether intraoperative support variables (durations of crossclamp, total support, total CPB, and circulatory arrest) and, in the case of the Bayley Scales, family social class ${ }^{13}$ independently predicted outcomes or served as confounders of the effect of hematocrit level on outcomes. Other tests for association used analysis of variance or Spearman rank correlation coefficients. All $P$ values are 
TABLE 1. Preoperative and operative characteristics of 271 patients undergoing hypothermic CPB in infant heart surgery

\begin{tabular}{|c|c|}
\hline Variable & Mean \pm SD \\
\hline \multicolumn{2}{|l|}{ Preoperative characteristics } \\
\hline Birth weight $(\mathrm{kg})$ & $3.4 \pm 0.6$ \\
\hline Gestational age (wk) & $39.1 \pm 1.5$ \\
\hline Apgar score at $5 \mathrm{~min}$ & $8.6 \pm 0.9$ \\
\hline Age at operation (d), median (range) & $41(1-269)$ \\
\hline Sex $(\%$ male $)$ & 65 \\
\hline Race (\% nonwhite) & 20 \\
\hline \multicolumn{2}{|l|}{ Diagnosis, n (\%) } \\
\hline \multicolumn{2}{|l|}{ D-TGA group } \\
\hline D-TGA, IVS & $61(23)$ \\
\hline D-TGA, VSD & $47(17)$ \\
\hline \multicolumn{2}{|l|}{ TOF group } \\
\hline TOF & $71(26)$ \\
\hline TOF, PA & $7(3)$ \\
\hline Truncus arteriosus & $13(5)$ \\
\hline \multicolumn{2}{|l|}{ VSD group } \\
\hline VSD & $65(24)$ \\
\hline Complete common AV canal & $7(3)$ \\
\hline Ever intubated $(\%)$ & 38 \\
\hline $\begin{array}{l}\text { Overall neurologic examination result } \\
\text { ( } \% \text { abnormal) }\end{array}$ & 62 \\
\hline \multicolumn{2}{|l|}{ Operative characteristics } \\
\hline Hematocrit level at onset of low-flow CPB (\%) & $26.5 \pm 5.3$ \\
\hline $16 \%-20 \%, \mathrm{n}(\%)$ & $37(14)$ \\
\hline $21 \%-25 \%$ & $84(31)$ \\
\hline $26 \%-30 \%$ & $85(31)$ \\
\hline $31 \%-35 \%$ & $49(18)$ \\
\hline $36 \%-39 \%$ & $16(6)$ \\
\hline Crossclamp time (min) & $65 \pm 26$ \\
\hline Total support time (min) & $108 \pm 37$ \\
\hline Total CPB time (min) & $100 \pm 32$ \\
\hline Duration of circulatory arrest (min) & $8 \pm 13$ \\
\hline None, n (\%) & $153(56)$ \\
\hline $1-10$ & $53(20)$ \\
\hline $11-30$ & $43(16)$ \\
\hline $31-44$ & $16(6)$ \\
\hline$\geq 45$ & $6(2)$ \\
\hline
\end{tabular}

$C P B$, Cardiopulmonary bypass; $S D$, standard deviation; $D-T G A$, dextrotransposition of the great arteries; IVS, interventricular septum; VSD, ventricular septal defect; TOF, tetralogy of Fallot; $P A$, pulmonary atresia; $A V$, atrioventricular.

2-tailed and we selected a level of significance of .05 for hypothesis testing.

\section{Results}

Descriptive statistics on preoperative and operative characteristics of the 271 combined study patients are included in Table 1. Hematocrit levels at the onset of low-flow CPB ranged from $16 \%$ to $39 \%$ and were not associated with diagnostic group $(26.6 \% \pm 5.2 \%$, mean $\pm \mathrm{SD}$, for the
TABLE 2. Perioperative and 1-year outcomes of 271 patients undergoing hypothermic CPB in infant heart surgery

\begin{tabular}{|c|c|c|}
\hline Variable & $\mathbf{n}$ & Mean \pm SD \\
\hline \multicolumn{3}{|l|}{ Perioperative outcomes } \\
\hline Intraoperative fluid balance (mL) & 244 & $401 \pm 259$ \\
\hline $\begin{array}{l}\text { Intraoperative blood products (mL), } \\
\text { median (range) }\end{array}$ & 270 & $208(25-1383)$ \\
\hline $\begin{array}{l}\text { Serum lactate at } 60 \text { min after CPB } \\
\text { (mmol/L) }\end{array}$ & 239 & $2.9 \pm 1.6$ \\
\hline $\begin{array}{l}\text { Nadir of cardiac index }\left(\mathrm{L} \cdot \mathrm{min}^{-1} \text {. }\right. \\
\left.\mathrm{m}^{-2}\right)\end{array}$ & 102 & $3.1 \pm 1.1$ \\
\hline $\begin{array}{l}\text { Postoperative blood products }(\mathrm{mL}) \text {, } \\
\text { median (range) }\end{array}$ & 271 & $111(0-3551)$ \\
\hline \multicolumn{3}{|l|}{ One-year outcomes } \\
\hline PDI & 215 & $86.2 \pm 15.7$ \\
\hline MDI & 216 & $93.9 \pm 12.4$ \\
\hline
\end{tabular}

$C P B$, Cardiopulmonary bypass; $S D$, standard deviation; $P D I$, Psychomotor Development Index; MDI, Mental Development Index.

D-TGA group; $26.2 \% \pm 5.5 \%$ for the tetralogy of Fallot [TOF] group; and $26.9 \% \pm 5.2 \%$ for the ventricular septal defect [VSD] group; analysis of variance $P=.64$ ) or with duration of crossclamp (Spearman rank correlation $r=$ 0.04, $P=.53)$, total support $(r=0.03, P=.61)$, total CPB $(r=0.03, P=.68)$, or circulatory arrest $(r=0.004, P=$ $.95)$. One infant $(0.4 \%)$ died within 1 month of the operation.

\section{Perioperative Outcomes}

Descriptive statistics on perioperative and 1-year outcomes are included in Table 2. We assessed the relationship of hematocrit level at the onset of low-flow CPB to intraoperative fluid balance. In generalized additive models adjusting for diagnostic group and year of operation, we found no significant departures from linearity $(P=.83)$. In linear regression models adjusting for diagnostic group and year of operation, increasing hematocrit level was significantly associated with decreasing fluid balance; each 1-point increase in hematocrit level was associated with an $11.8-\mathrm{mL}$ decrease in intraoperative fluid balance $(95 \%$ confidence interval $[\mathrm{CI}]=5.5-18.1 \mathrm{~mL} ; P<.001$, Figure $1, A)$. Independent predictors of higher intraoperative fluid balance included diagnostic group (D-TGA highest, TOF intermediate, VSD lowest; $P<.001$ ), later year of operation $(P<.001)$, and longer crossclamp, total support, and total CPB times $(P<.001$ for each), but not duration of circulatory arrest. Inclusion of these support times in the multivariable linear model further increased the strength of the relationship of higher hematocrit at onset of low-flow CPB to lower intraoperative fluid balance.

Although higher fluid balance was significantly associated with longer time until endotracheal intubation, intensive care unit discharge, and hospital discharge, hematocrit 

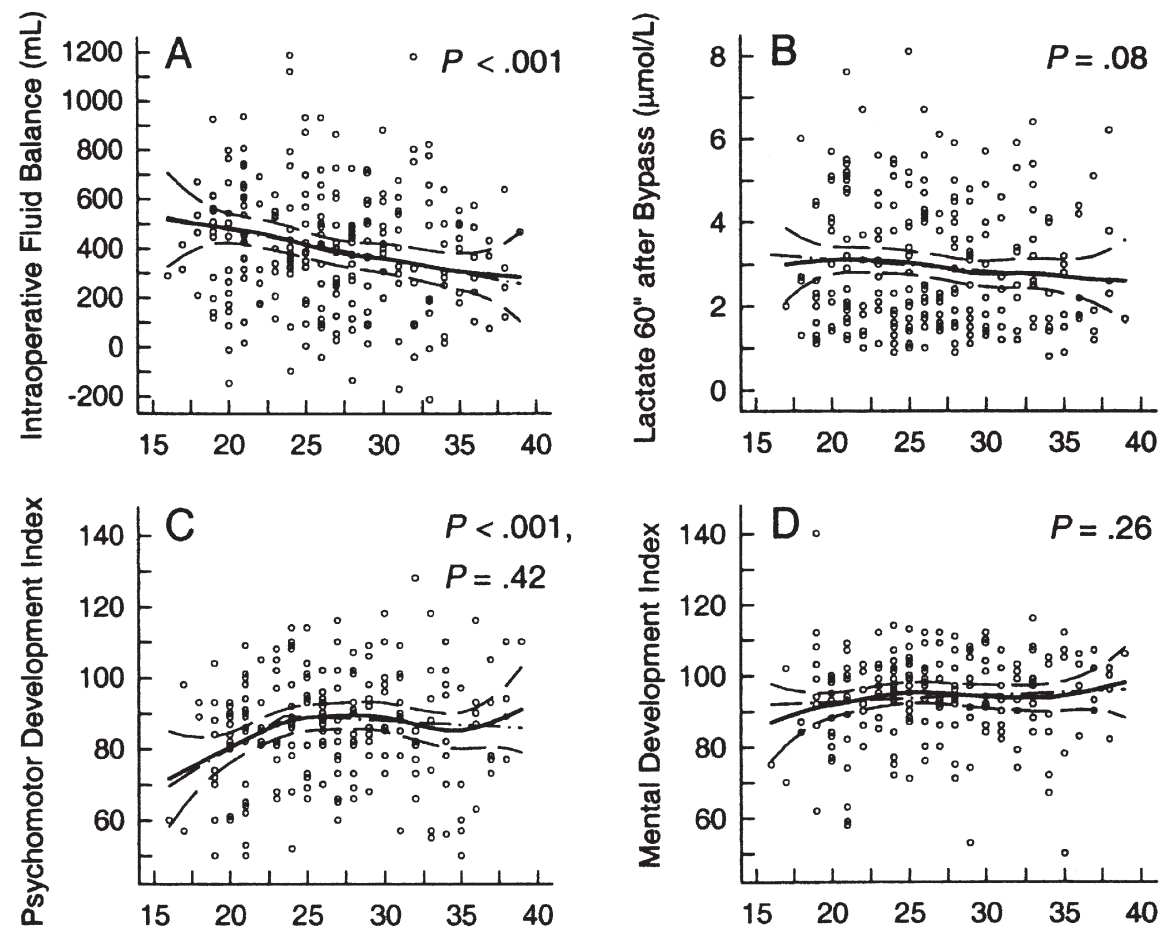

Hematocrit at Onset of Low Flow (\%)

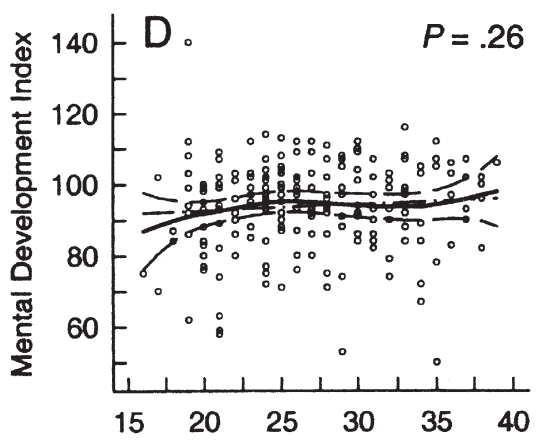

Hematocrit at Onset of Low Flow (\%)
Figure 1. Scatterplots of outcomes as a function of hematocrit level at the onset of low-flow CPB. The panels represent intraoperative fluid balance $(A)$, serum lactate levels at $\mathbf{6 0}$ minutes after cessation of CPB (B), Psychomotor Development Index (C), and Mental Development Index (D). The solid lines were derived through generalized additive models, with pointwise 95\% confidence limits for hematocrit effects denoted as dashed lines. The dotdash lines denote the fitted linear regression models. In the case of Psychomotor Development Index, the dotdash line denotes the fitted piecewise linear regression model, based on the optimal cut point of $23.5 \%$ hematocrit. The $\boldsymbol{P}$ values assess the strength of the relationship between hematocrit on outcome in adjusted linear regression models. For Psychomotor Development Index, the top $\boldsymbol{P}$ value corresponds to the effect before and the bottom $P$ value corresponds to the effect after the optimal cut point of $23.5 \%$ hematocrit. All models adjust for diagnostic group and models for intraoperative fluid balance also adjust for year of operation. Generalized additive model fits and linear regression model fits are plotted for average values of these adjustment factors. level at the onset of low-flow CPB was not an independent predictor of these measures of prolonged recovery in multivariable models adjusting for diagnosis or year of operation. Furthermore, analyses within diagnostic groups also did not show associations between hematocrit level and length-of-stay variables.

We assessed the relationship of hematocrit level at the onset of low-flow CPB to serum lactate levels at 60 minutes after cessation of CPB. In generalized additive models adjusting for diagnostic group, we found no significant departures from linearity $(P=.73)$. In linear regression models adjusting for diagnostic group, higher hematocrit level was marginally associated with lower lactate level; each 1-point increase in hematocrit level was associated with a 0.027 $\mathrm{mmol} / \mathrm{L}$ decrease in serum lactate levels at 60 minutes after cessation of CPB $(95 \% \mathrm{CI}=-0.003-0.057 \mathrm{mmol} / \mathrm{L} ; P=$ .08 , Figure $1, B)$. Independent predictors of higher serum lactate included diagnostic group (D-TGA highest, TOF intermediate, VSD lowest; $P<.001$ ), longer total support time $(P=.009)$, and longer duration of circulatory arrest $(P<.001)$. Inclusion of these support times in the multivariable linear model increased the strength of the relationship of higher hematocrit level at onset of low-flow CPB to lower serum lactate levels.
Nadir of cardiac index was measured in only 102 patients. Adjusting for diagnostic group, we found no significant departures from linearity of the effects of hematocrit level on nadir of cardiac index $(P=.44)$ and no significant linear effects of hematocrit level on nadir of cardiac index $(P=.24)$. The only independent predictor of nadir of cardiac index was diagnostic group (D-TGA lowest, TOF intermediate, and VSD highest; $P<.001)$.

We measured the use of blood products intraoperatively and in the postoperative period. Adjusting for diagnostic group and, for intraoperative blood products only, year of operation, there were no significant departures from linearity and no significant linear effects in the relationships of hematocrit level to intraoperative or postoperative blood product use. Independent predictors of higher intraoperative blood products included diagnostic group (D-TGA highest, TOF intermediate, VSD lowest; $P<.001$ ), later calendar year of operation $(P=.004)$, and longer crossclamp, total support, and total CPB times $(P<.001$ for each), but not duration of circulatory arrest. Independent predictors of higher postoperative blood products included longer crossclamp, total support, total $\mathrm{CPB}$, and circulatory arrest times $(P<.001$ for each). Inclusion of these support times in the multivariable linear models did not affect the lack of sig- 
nificant association between hematocrit levels and postoperative blood products.

\section{One-year Outcomes}

We assessed the relationship of hematocrit at the onset of low-flow CPB to PDI scores at age 1 year. In generalized additive models adjusting for diagnostic group, we found significant departures from linearity $(P=.008)$. Evaluation of piecewise linear models over a grid of hematocrit cut points led to an optimal cut point of $23.5 \%$, with a $95 \%$ CI from $21.0 \%$ to $29.5 \%$. In a piecewise linear model, each 1-point increase in hematocrit up to $23.5 \%$ was associated with a 2.6-point increase (slope) in PDI scores $(95 \% \mathrm{CI}=$ $1.1-4.2 ; P<.001)$. However, increasing hematocrit level above $23.5 \%$ was not significantly associated with PDI scores (slope $=-0.2,95 \% \mathrm{CI}-0.8-0.3 ; P=.42$ ), and PDI scores had effectively reached a plateau by a hematocrit level of $23.5 \%$ (Figure 1,C). The two slopes (ie, below and above hematocrit levels of $23.5 \%$ ) were significantly different $(P=.003)$. Generalized additive models showed no significant evidence of departure from the piecewise linear model $(P=$ .32). Choice of other cut points led to similar inferences. Intraoperative support times and social class did not serve as significant independent predictors of PDI scores.

We assessed the relationship of hematocrit at the onset of low-flow CPB to MDI scores at age 1 year. Adjusting for diagnostic group, we found no significant departures from linearity of the effects of hematocrit on MDI scores $(P=.34)$ and no significant linear effects of hematocrit on MDI $(P=$ .26) (Figure $1, D)$. Higher family social class was independently associated with higher MDI scores $(P<.001)$.

\section{Discussion}

We found that PDI scores at age 1 year varied nonlinearly with hematocrit levels, with significantly increasing scores up to $23.5 \%$ hematocrit and a plateau effect beyond $23.5 \%$ hematocrit, based on a piecewise linear model. Lower hematocrit levels were significantly associated with higher fluid balance and marginally associated with higher serum lactate levels at 60 minutes after $\mathrm{CPB}$, but not associated with nadir of cardiac index in the first 24 hours, blood products given, or MDI scores. It may be appropriate to target hematocrit level at the onset of low-flow CPB to be at least $24 \%$, inasmuch as lower hematocrit levels are associated with worse PDI scores and a tendency for higher lactate levels, whereas higher hematocrit levels were not associated with adverse events or outcomes.

The correlation/linear regression methods used in previous studies, which force a linear fit to the relationship between hemodilution and outcomes, are problematic if there is a nonlinear trend between these variables. In particular, the overall slope or correlation is a weighted average of the slopes or correlations over the whole range of hematocrit values, which could underestimate the effects of hemodilution over certain ranges of hematocrit values if there are nonlinear trends. Our use of nonparametric regression smoothing and piecewise linear regression provided an opportunity to identify potential cut points in these associations.

We found no relationship of hematocrit level at the onset of low-flow CPB with usage of blood products in the operating room or during the postoperative period. Animal models of hematocrit strategy have shown that hemodilution not only reduces oxygen-carrying capacity, but also reduces osmolality, viscosity, and perfusion pressure, as well as diluting platelets and coagulation factors. ${ }^{4,14}$ The lack of association of hematocrit levels with blood product use may reflect improved hemostasis with higher hematocrit levels.

Laboratory studies have demonstrated the important interaction of hematocrit with temperature, $\mathrm{pH}$ strategy, and duration of circulatory arrest or very reduced flow. ${ }^{15,16}$ Specifically, animal models have demonstrated that it is possible to compensate for the reduced oxygen-carrying capacity of low hematocrit by increasing oxygen availability by the $\mathrm{pH}$-stat strategy or by increasing flow rate, reducing duration of circulatory arrest, or reducing temperature. In both hematocrit trials, we used the $\mathrm{pH}$-stat strategy, deep hypothermia in many patients, and minimal periods of extended circulatory arrest or very low-flow CPB. These aspects of the intraoperative milieu, together with the potential inclusion of some infants with undetected genetic syndromes, may have limited our power to detect effects of hematocrit on some outcomes.

Our analyses should be interpreted in light of several additional limitations. Both hematocrit trials were conducted in a single center and included only infants undergoing reparative cardiac surgery for congenital heart defects involving two ventricles without aortic arch obstruction. These aspects of the study could limit generalizability to other centers and congenital heart lesions. Furthermore, although we excluded infants with known or suspected genetic or congenital anomalies, the study protocols did not require fluorescent in situ hybridization studies to exclude genetic disorders; the inadvertent inclusion of some children with genetic disorders could have diminished the effect of hematocrit level on 1-year neurodevelopmental outcomes.

In summary, we found that the effect of hemodilution level on PDI scores is nonlinear, with improving scores with increases in hematocrit level at the onset of low-flow CPB up to approximately $24 \%$, followed by a plateau. Increasing hematocrit levels were also marginally related to a reduction in serum lactate levels 60 minutes after cessation of CPB. This study cannot ascertain a universally optimal hematocrit level because of potential influences of patient characteristics and other intraoperative factors, but at Children's Hos- 
pital Boston, we try to ensure that hematocrit level will be above $25 \%$ at commencement of CPB and during CPB and rewarming. Future multicenter investigations should ascertain late neurodevelopmental status after infant cardiac surgery and study other populations, including adults, to help to clarify optimal hematocrit levels during CPB.

We thank our perfusionists: Willis G. Gieser, CCP, Robert A. LaPierre, BS, CCP, Robert J. Howe, BS, CCP, Gregory S. Matte, CCP, and William L. Regan; our nursing staff in the Cardiac Intensive Care Unit for assistance with adherence to the protocols; Ludmila Kyn for database and statistical programming; Donna Donati, Donna Duva, and Lisa-Jean Buckley for data management; Kathleen Alexander for project coordination; and families and children in the cohort for their time and effort.

\section{References}

1. Neptune WB, Bougas JA, Panico FG. Open heart surgery without the need for donor blood priming in the pump oxygenator. New Engl J Med. 1960;263:111-5.

2. Cooper JR, Slogoff S. Hemodilution and priming solutions for cardiopulmonary bypass. In: Gravlee GP, Davis RF, Utley JR, editors. Cardiopulmonary bypass principles and practice. Baltimore, MD: Williams and Wilkins; 1993. p. 124-37.

3. Gold JP, Charlson ME, Williams-Russo P, Szatrowski TP, Peterson JC, Pirraglia PA, et al. Improvement of outcomes after coronary artery bypass: a randomized trial comparing intraoperative high versus low mean arterial pressure. J Thorac Cardiovasc Surg. 1995;110:1302-11.

4. Shin'oka T, Shum-Tim D, Jonas RA, Lidov HG, Laussen PC, Miura T, et al. Higher hematocrit improves cerebral outcome after deep hypothermic circulatory arrest. J Thorac Cardiovasc Surg. 1996;112: 1610-21.
5. Sungurtekin H, Cook DJ, Orszulak TA, Daly RC, Mullany CJ. Cerebral response to hemodilution during hypothermic cardiopulmonary bypass in adults. Anesth Analg. 1999;89:1078-83.

6. Jonas RA, Wypij D, Roth SJ, Bellinger DC, Visconti KJ, du Plessis AJ, et al. The influence of hemodilution on outcome after hypothermic cardiopulmonary bypass: results of a randomized trial in infants. J Thorac Cardiovasc Surg. 2003;126:1765-74.

7. Newburger JW, Jonas RA, Soul J, Kussman BD, Bellinger DC, Laussen PC, et al. Randomized trial of hematocrit $25 \%$ versus $35 \%$ during hypothermic cardiopulmonary bypass in infant heart surgery. J Thorac Cardiovasc Surg. 2008; 135:347-54

8. Bayley N. Bayley scales of infant development. 2nd ed. San Antonio, TX: The Psychological Corporation;1993.

9. Hastie TJ, Tibshirani KJ. Generalized additive models. London: Chapman and Hall; 1990.

10. SAS/STAT 9.1 user's guide. Cary (NC): SAS Institute Inc; 2004.

11. Casella G, Berger RL. Statistical inference. 2nd ed. Belmont (CA): Duxbury Press; 2002.

12. Wypij D, Newburger JW, Rappaport LA, duPlessis AJ, Jonas RA, Wernovsky G, et al. The effect of duration of deep hypothermic circulatory arrest in infant heart surgery on late neurodevelopment: the Boston Circulatory Arrest Trial. J Thorac Cardiovasc Surg. 2003;126: 1397-403.

13. Hollingshead A. Four factor index of social status. New Haven, CT; Unpublished manuscript; 1975.

14. Shin'oka T, Shum-Tim D, Laussen PC, Zinkovsky SM, Lidov HG, du Plessis A, et al. Relative roles of colloid oncotic pressure and hematocrit in determining outcome after deep hypothermic circulatory arrest. Ann Thorac Surg. 1998;65:155-64.

15. Sakamoto T, Zurakowski D, Duebener LF, Lidon HG, Holmes GL, Hurley RJ, et al. Interaction of temperature with hematocrit level and $\mathrm{pH}$ determines safe duration of hypothermic circulatory arrest. $J$ Thorac Cardiovasc Surg. 2004;128:220-32.

16. Anttila V, Hagino I, Zurakowski D, Iwata Y, Duebener L, Lidov HG, et al. Specific bypass conditions determine safe minimum flow rate. Ann Thorac Surg. 2005;80:1460-7.

\section{JTCVS On-Line Manuscript Submission and Review}

The Journal of Thoracic and Cardiovascular Surgery requires authors and reviewers to submit all new and revised manuscripts and reviews via Editorial Manager. Point your browser to http://jtcvs.editorialmanager.com, log in as author or reviewer (as appropriate), and follow the instructions provided.

To retrieve your username and password, click "Forget your password?" on the Editorial Manager log-in page.

If you have questions or experience problems uploading your manuscript or review, please contact the editorial office:

Telephone: 978-299-4516

E-mail: rwalther@prri.com 